\title{
A Study of Geographic Adaptive Fidelity Routing Protocol in Wireless Sensor Network
}

\author{
Jitender Grover ${ }^{1}$, Shikha ${ }^{2}$, Mohit Sharma ${ }^{3}$ \\ ${ }^{1,2,3}$ (Department of Computer Science \& Engineering, M. M. University, Sadopur, Ambala, India)
}

\begin{abstract}
The Energy consumption is a great challenge in the field on Wireless Sensor Network (WSN). It affects the performance of the whole network. There are two basic functions of nodes in the WSN. First is to collect the information from other sensor nodes and second is to pass the information to the base station. A variety of routing protocols have been proposed to reduce the energy consumption of nodes and increase the network lifetime. One of the most popular energy efficient routing protocol is Geographic Adaptive Fidelity $(G A F)$. This is the location based routing protocol which uses the nodes location information to transfer the data. This reduces the use of energy by turning off the radio of some nodes which share the same functionalities. This paper focuses on the GAF protocol and its working. Also this paper reviews the variety of new versions based on GAF protocol to make it better.
\end{abstract}

Keywords: Energy Efficiency, Location, GAF, WSN.

\section{Introduction}

A Wireless Sensor Network (WSN) is a network of large amount of sensor nodes which are densely deployed in an ad-hoc manner. Due to the small size and light weight they are easily deployed in an unreached area. Sensor node is a device which is programmed to perform the task of sensing, data processing and communication with the help of power. The two basic functions of sensor networks are collect the sensed information from nodes and transmit it to the base station either directly or through some gateway. Base station is generally fixed. WSN is used in a number of applications such as military, tracking, environmental monitoring and inventory control. The basic requirement is that the nodes will remain active for a long period. Sensor nodes need some amount of energy to receive or transmit the data. Lack of energy affects the performance of the network.

There are various issues in WSN but the most challenging issue is to save the energy of node. After the deployment of sensors it is very difficult or impossible to replace or recharge the battery. The depletion of energy may results into link failure due to which the purpose of application may fail. A variety of routing protocols have been designed which uses the different techniques to minimize the energy consumption of nodes and increase the network lifetime. Some techniques for energy minimization are [1]:

Data Reduction: Maximum energy of node is used at the time of processing and transmitting the data. To save some energy the amount of data can be reduced by using some techniques. Mostly used techniques to reduce the data are data compression or data aggregation.

Protocol Overhead Reduction: A lot of energy is wasted because of protocol overhead. Reduction in the overhead minimizes the energy consumption and increases protocol efficiency. Basic technique is to avoid unnecessary retransmissions by optimized flooding.

Topology Control: A reduced topology is to be formed which will save the energy of nodes and does not affect the connectivity of network. This can be achieved by adjusting the transmission power of sensor nodes.

Energy Efficient Protocols: Routing protocols are designed with the aim to overcome the energy challenge of WSN. Nodes having high energy should participate in transmission and avoid low energy nodes. Energy efficient routing protocols increase the lifetime of whole network.

Sleep/Active Scheduling: Idle nodes also use some amount of energy. In sleep/active scheduling scheme, unnecessary idle nodes enters into sleep mode by turning radio off. Only active nodes will participate in data transmission and processing. This saves the energy of idle nodes.

In WSN, the energy consumption is directly proportional to the range i.e. there is more energy consumption if the range is large [2]. This is calculated from the relation

$$
\mathrm{E}=\mathrm{kd}^{\mathrm{n}}
$$

Where $\mathrm{E}$ is energy consumption, $\mathrm{k}$ is a constant, $\mathrm{d}$ is the range of communication and $2<\mathrm{n}<4$.

This paper studies the most popular energy aware routing protocol known as Geographic Adaptive Fidelity (GAF). This is a location based routing protocol. This uses the technique of sleep/active schedule. 


\section{GAF (Geographic Adaptive Fidelity)}

GAF is a location based and an energy aware routing protocol in WSN. Nodes use location information through any system like GPS, received radio signal strength etc to locate itself along with its nearest neighbors. Nodes consume energy while transmitting data i.e. at the time of sending as well as receiving data. In the idle state some amount of energy is used but it is less in comparison to active state. Energy used in the idle mode can be saved by turning off the radios.

In GAF, the whole network is divided into a virtual grid. The proper size of grid is important as it directly affects the network connectivity. If the grid size is large then it is difficult to connect the whole network by activating just one node per grid. The size of grid (r) is based on the concept that any node can communicate with any other node present in the neighboring grid. The grid size $r$ is

where $\mathrm{R}$ is the radio range [3].

$$
\mathrm{r} \leq \mathrm{R} / \sqrt{ } 5
$$

Figure 1 show the network divided in virtual grid. [3] [4]. There are five nodes in the network. Node 1 can communicate with Node 5 with the help of sending the data to any of the intermediate nodes namely 2,3 and 4. To conserve the energy two nodes ( 3 and 4 ) from the same grid go to sleep mode as all the nodes in the same grid are considered to be of same functionalities. Now node 1 sends data to node 2 , which further transmits it to node 5 . This is known as routing fidelity where both nodes 1 and 5 are communicating using only one efficient node as their routing partner and other intermediate nodes go into sleep mode.

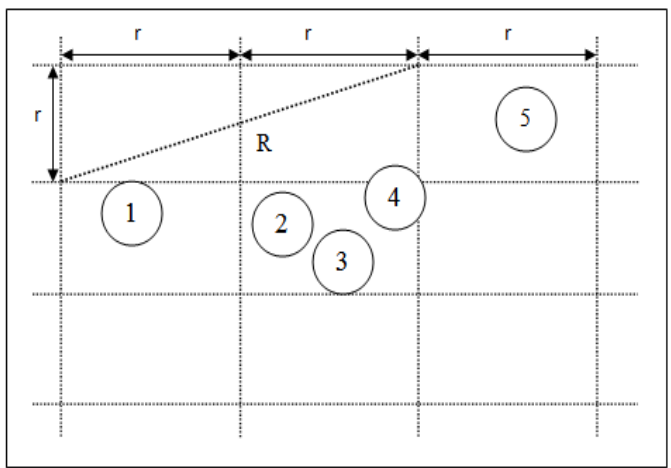

Fig 1: Virtual Grid in GAF

\section{Transition States in GAF}

The different transition states used in GAF are sleeping state, Discovery state and Active state [4] [5]. Only one node per grid is in the active state and all others are in sleeping state in order to save the energy. The selection of node to be active depends on the residual energy of the nodes. Figure 2 shows the different transition states in GAF protocol.

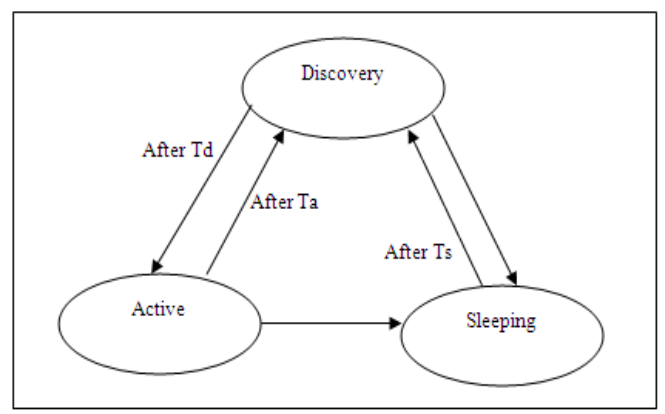

Fig 2: Transition States in GAF Protocol

Initially every node starts with the discovery state and then enters into active or sleep state. The node in the active state is responsible for monitoring and reporting data to the sink or BS on behalf of the nodes in the grid. How the state is changed in GAF protocol is described below.

\section{- State Transition from Discovery to Active}

Each node exchanges discovery messages to find its equivalent nodes. All the nodes of the same grid are equivalent. A discovery message contains some information about the node i.e. node id (its current location), grid id, node state and energy level (enat: estimated node active time). After predefined time Td the node enters into the active state if it does not receive any other discovery message. 


\section{- State Transition from Discovery to Sleeping}

In the discovery state, if node receives any other discovery message from another node having higher energy level than a node enters into the sleep state. At a particular time only one node is in active state else are in sleep state. In order to keep the routing fidelity, sleeping neighbors adjust their sleeping time (Ts) accordingly. One of the sleeping nodes becomes active much before the leaving time of the active node expires.

\section{- State Transition from Active to Sleeping}

Ta is a time which shows that for how long a node will stay in an active state. After Ta, if another node having high energy is present in the grid then current active node will enters into a sleep state.

\section{- State Transition from Sleeping to Discovery}

The node has to complete the sleep time Ts before wake up and to enter into the discovery phase. After Ts node again enters into a discovery phase and if it has highest energy level then enters into active state otherwise re-enters into sleep state.

\section{- State Transition from Active to Discovery}

A node enters into the discovery phase after a predefined time Ta and rebroadcasts the discovery message for time $\mathrm{Td}$. If it receives a message from another node having higher residual energy then it enter into sleep state else re-enters into active state.

\section{Improvements Over GAF}

A lot of research is performed on the basis of GAF protocol. Researchers modified GAF into new protocol to make the network performance better. Some of the proposed techniques are described in this paper.

\section{A. Hierarchical Geographical Adaptive Fidelity (HGAF)}

Hierarchical Geographic Adaptive Fidelity (HGAF) is proposed to save the power of the nodes which increases the lifetime of whole network by the authors in [6]. HGAF uses a layered structure. After dividing the network into grids like GAF, HGAF divides each grid or cell into squared sub-cells. A node is active in one of these square sub-cells. The sub-cell having an active node is known as active sub-cell. All other nodes of cell are in sleep mode. Communication takes place between active nodes. This reduces the distance between two farthest nodes in adjacent cells. The numbers of sub-cells in each cell of HGAF are $\mathrm{N}^{2}$. Size of each cell is $\mathrm{r}^{*} \mathrm{r}$ and the size of each sub-cell is $\mathrm{d}^{*} \mathrm{~d}$. Figure 3 shows the layered structure of HGAF with $\mathrm{N}=3$ and $\mathrm{AB}$ is the distance between two farthest nodes which is smaller than communication range R in GAF. This saves much more battery power because it decreases the number of active nodes.

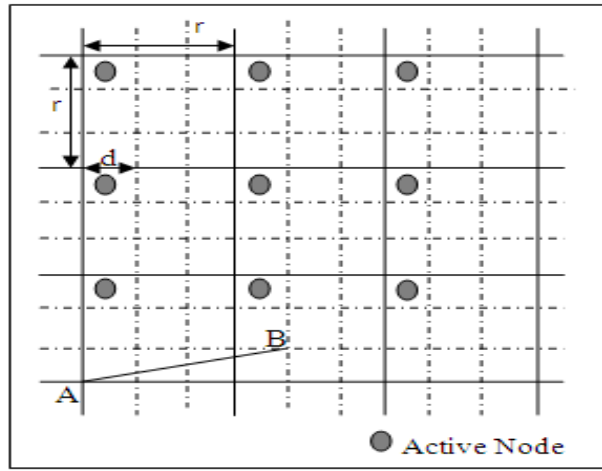

Fig 3: Layered Structure in HGAF.

For the purpose of load balancing, the active sub-cells are rotated periodically. After the predefined time active sub-cells rotate the position sequentially as in Figure 4. Rotation scheme is same in all cells. Results in [6] shows that HGAF performs better than GAF in case of energy efficiency and packet delivery ratio. Also when there is high node density and a cell is further divided into four sub cells then the lifetime of network is increased $200 \%$ in HGAF comparison to GAF. 


\begin{tabular}{|c|c|c|c|}
\hline 1 & 2 & 1 & 2 \\
\hdashline 4 & 3 & 4 & 3 \\
\hline 1 & 2 & 1 & 2 \\
\hdashline 4 & 3 & 4 & 3 \\
\hline
\end{tabular}

Fig 4: Rotation Scheme in HGAF.

\section{B. Extended HGAF (eHGAF)}

In Extended HGAF, the cell boundary moves in place of rotating the active sub-cell. This scheme works on the concept that active sub-cell lies in the centre of the cell. Due to this the distance between active sub-cell and other sub-cells in the cells is smaller than HGAF because in HGAF, any sub-cell become an active sub-cell but in eHGAF only the center sub-cell will become active. Figure 5 shows the working of eHGAF. Figure 5(a) shows the current state of the network in eHGAF and Figure 5(b) shows the state change by moving cell boundary after the predefined time.

In eHGAF, there are two cases which are explained below [6]:

Case I: When $\mathrm{N}$ is an Odd Number

In the case when $\mathrm{N}$ is an odd number i.e. $\mathrm{N}=2 \mathrm{n}-1$ where $\mathrm{n}=1,2,3 \ldots$, then it is easy to select a centre sub-cell to become an active sub-cell.

Case II: When $\mathrm{N}$ is an Even Number

In the case when $\mathrm{N}$ is an even number i.e. $\mathrm{N}=2 \mathrm{n}$ where $\mathrm{n}=1,2,3 \ldots \ldots$, then there is more than one sub-cell sharing the center point of the cell. Then in this case an arbitrary sub-call lying next to the centre point is active.

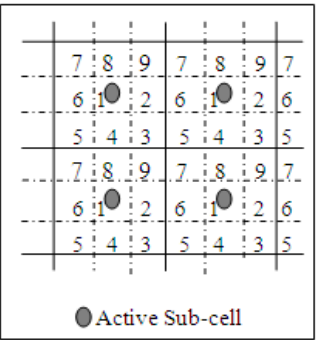

(a)

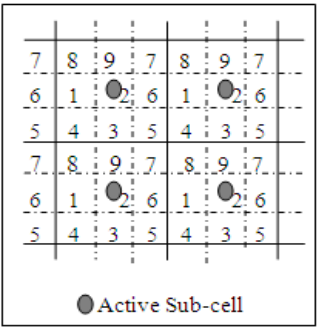

(b)

(a) Cell is divided into sub-cells and active sub-cell lies in the centre.

(b) After time 't' boundaries of cell moves but sub-cell remains in the center.

Fig 5: eHGAF Structure.

The extended version of HGAF i.e. eHGAF (extended HGAF) is further improved to save the energy consumption by dividing the sensor field in an efficient way and increase the lifetime of network [7]. It works on two concepts:

- Cell enlargement by changing cell shape to triangle cells.

- Cell enlargement by reducing edges.

\section{GAF with COnnectivity-awareness (GAF\&Co)}

A topology management protocol known as GAF with COnnectivity-awareness (GAF\&Co) is proposed to maintain the connectivity of a network and to avoid routing holes. This also helps in energy consumption by activating an energy-saving mode for the redundant nodes. This protocol is derived from GAF protocol. In GAF fewer nodes are used in routing process which results in reduced connectivity of the network and number of routing holes increases. To avoid the problem of local minimums GAF\&Co divides the network into hexagonal cells in place of virtual grids. To check whether a node can be a local minimum the TENT rule is applied. [8] According to TENT rule an angle between node and its angular adjacent neighbor node pair is not more than $2 \pi / 3$. 


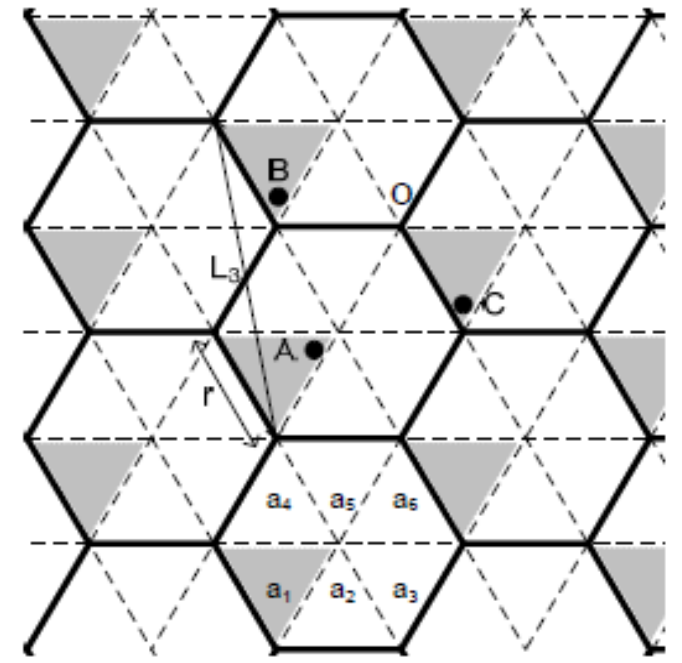

Fig 6: Hexagonal Cell Division in GAF\&Co Protocol.

In Figure 6, the network is divided into hexagonal cell and each cell is further divided into 6 triangular sub-cells (a1, a2, a3, a4, a5, a6). Grey areas in network shows the active sub-cell which contains an active node. Sub-cells are selected with the same relative position in each cell. An active node is selected by applying same algorithm as in GAF. By using hierarchical sub-cells the size of the cell expands which reduces the number of active nodes in network. Table 1 shows the maximum cell size for different GAF based protocols. The communication range $\mathrm{R}$ of nodes in this protocol is: $\mathrm{R} \geq \mathrm{L}_{3}$

Table I: Maximum Cell Size for GAF Based Protocols

\begin{tabular}{|l|l|}
\hline Protocols & Max cell size \\
\hline GAF & $\frac{\mathbf{1}}{\mathbf{5}} \mathrm{R}^{2}$ \\
\hline HGAF & $\frac{\mathbf{1}}{\mathbf{2}} \mathrm{R}^{2}$ \\
\hline eHGAF & $\mathrm{R}^{2}$ \\
\hline eHGAF with triangle cells & $\frac{\mathbf{3} \sqrt{\mathbf{3}}}{\mathbf{4}} \mathrm{R}^{2}$ \\
\hline eHGAF with two cell types & $\sqrt{3} \mathrm{R}^{2}$ \\
\hline GAF\&Co. & $\frac{\mathbf{1}}{\mathbf{7}} \mathrm{R}^{2}$ \\
\hline
\end{tabular}

\section{Dynamic-division GAF (DGAF)}

The division of network and selecting a proper cluster head also affects the overall performance of the network. The division of network is based on the calculation that according to the position information node belongs to which grid. The proposed protocol is known as DGAF (Dynamic-division Geographical Adaptive Fidelity). The basic purpose of this algorithm is to find the optimum position of the cluster head. According to [9], the cluster head should be selected near the centre of their region because it consumes less energy in comparison to cluster head situated at the border of the region. The area is divided into grids as hexagons named as DGAF-6; or as squares named as DGAF-4. This protocol works in two steps which are as following:

\section{Step 1: Dividing into virtual grids}

Every node knows its position in the network. By the help of calculations node get the information to which grid it belongs to also its distance from the centre of the grid.

\section{Step 2: Selecting cluster head}

Selection of cluster head is done by the help of "Cost" factor. The node having lowest cost within the grid is selected as cluster head. Cluster heads are connected to each other for data transmitting. 


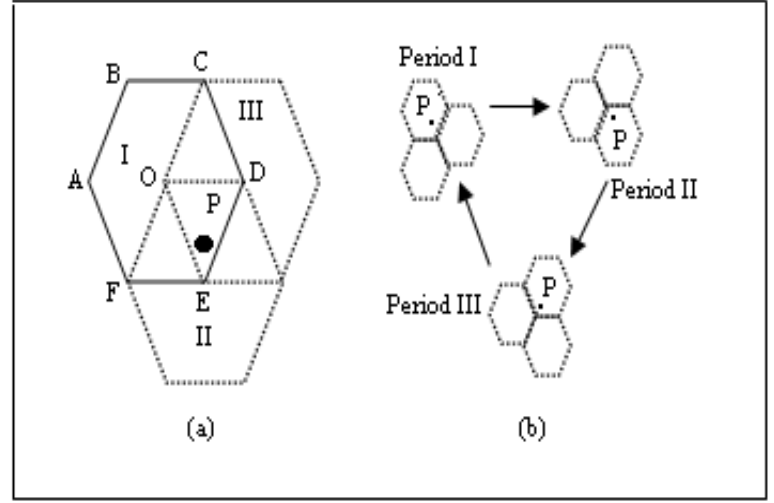

Fig 7: Dynamical Division of the Area

Figure 7 shows the concept used by DGAF. In Figure 7(a), P is situated at the border of virtual region I which is far away from the centre point. To place it nearer to the centre, divide the area by taking A, C and E (or $\mathrm{B}, \mathrm{D}$ and F) as a center point such as in region II, E is a centre and P is situated near E. Accordingly, the area is divided into virtual grids as in Fig 7(b) and repeat this after every 3 period. $\mathrm{P}$ will have high probability to be selected as a cluster head in $1 / 3$ time.

\section{E. GAF-HEX}

In GAF, network is divided into an equal size of square grid. In a square grid structure a node can reach to the adjacent grids only in vertical and horizontal directions but not in diagonal direction. This arise the problem of unreachable corners. Hexagonal grid structure (GAF-HEX) is proposed in this reference [10]. GAFHEX uses location information to find the position of nodes. According to GAF-HEX, every node in a cell can communicate with all the nodes of the adjacent cell. In square grid, each cell has only four cells are at one hop distance (two horizontal and two vertical) but in hexagonal grid there are six cells (all neighboring cells) at one hop distance.

GAF-C and GAF-E are two node mapping algorithms introduced for GAF-HEX. Figure 8 shows the mapping for GAF-C. It maps the nodes with reference node at centre. Figure 9 shows the mapping for GAF-E in which nodes are mapped with reference node at end of grid. GAF-HEX improves packet delivery ratio and throughput and energy consumption is almost same as GAF.

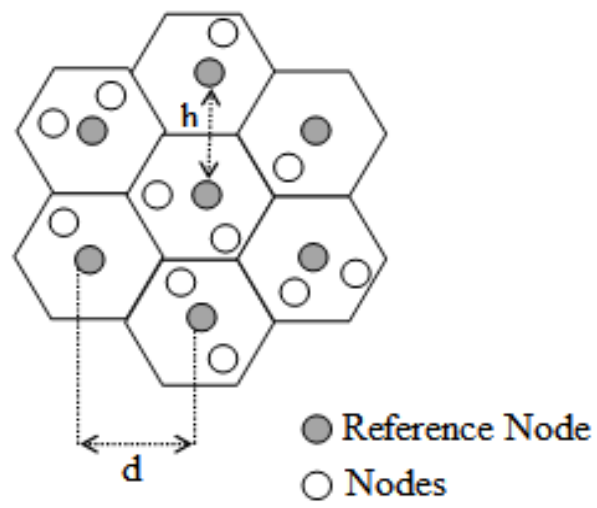

Fig 8: Node Mapping of GAF-C

The horizontal distance between two reference nodes is $\mathrm{h}$ and the vertical distance between the two is d. Each cell has one active node used for routing the data and other nodes are in sleep state. 


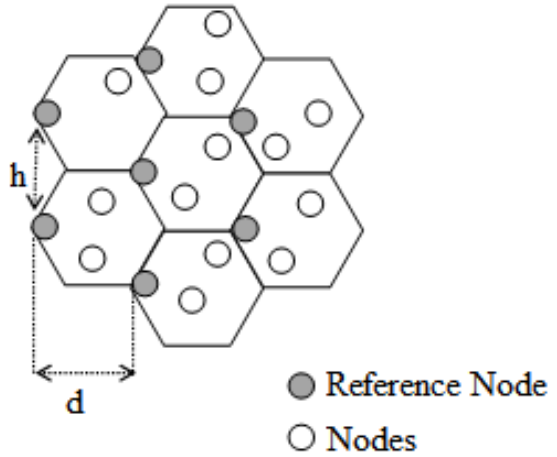

Fig 9: Node Mapping of GAF-E

\section{F. Sleep Doze Coordination Protocol}

To increase the lifetime of network by lowering the duty cycle of each node a protocol known as Sleep Doze Coordination (SDC) is proposed [11]. This uses a layered architecture of wireless sensor network. Each layer is broken into grids similar to GAF. According to SDC, there are two modes at each node:

a) "On" period \{Alert mode or Doze mode\}

b) "Off" period $\{$ Sleep mode $\}$

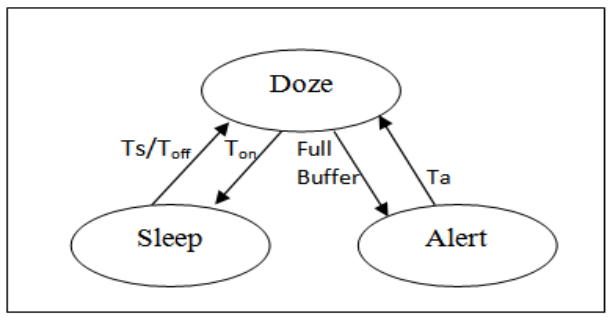

Fig 10: State Transitions in SDC Protocol

Idle listening state is known as doze state. One node per grid is in 'doze' state for a predefined time and rests are in sleep state. A node in the doze state enters into an active state when node buffer gets filled to its capacity by data messages from the lower layer. Transition states in SDC protocol are shown in Figure 10. In GAF, one node per grid is in active state. In SDC one node per grid should be doze state. Each node receives two types of messages. First, standard beacon messages which are processed when node transit to an active mode and other is urgent buffer messages which requires immediate processing hence node transit to active mode immediately.

SDC increases network lifetime by approximately $20 \%$ over GAF protocol. This is expensive because it needs a buffer. A new approach to the Sleep Doze Coordination protocol known as Lossless Sleep Doze Coordination (LSDC) is presented in [12]. This improves the lifetime of a network. Architecture used in this protocol is multilayered. In comparison to GAF, this sleep protocol has one node per grid in the doze state for some defined time. Figure 11 shows the state transitions in LSDC protocol. Nodes can sense and communicate with upper and lower layer sensors in active state. In interactive mode, node does not perform sensing. It only sends a message (standard beacon messages) to all its peer nodes and also receives reply. Sleepy state is a special case of doze state. After a doze state, node enters into sleepy state if all other nodes are sleeping and enters to sleep state whenever another node wakes up. The radios are turned off in sleep state and after a time Ts node enters into interactive state. Only one node is in doze state and rest is in sleep state. The node to be in doze state can be selected by different methods such as:

- Select randomly.

- Node having maximum battery power is selected.

- Node that has slept the longest ever is selected. 


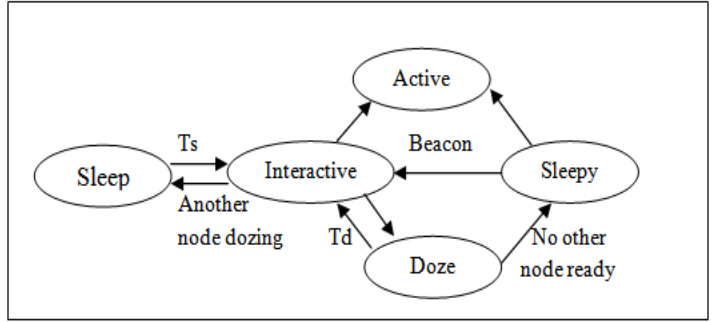

Fig 11: State Transitions in LSDC Protocol

\section{G. Some Other Improved Protocols}

The solution to conserve energy and increase the lifetime of the network is proposed in [13]. The proposed algorithm is based on Geographic Adaptive Fidelity (GAF) and Adaptive Self-Configuring Sensor Networks Topology (ASCENT). The main focus is on searching the exact location of the master node in a grid by sending test signals to all the nodes in a grid. The master node is selected at the centre of the grid and rest of the nodes enters to sleep state conserving energy. This helps to increase the lifetime of network in comparison to the traditional GAF in which node head is selected randomly.

To removes the coverage problem of GAF, a new algorithm Coverage-guaranteed Distributed Sleep/Wake Scheduling (CDSWS) is proposed [14]. According to CDSWS, clusters are formed and one node per cluster is in active state. There are three phases in CDSWS:

- Initialization phase: In this, packets are received and transmitted by the nodes.

- Cluster forming phase: In this, a large cluster will be formed by merging two clusters.

- Sleep/wake scheduling phase: One or two nodes per cluster is selected to be in active state and others are in sleep mode to save energy.

This algorithm has some advantages like coverage guarantee, algorithm efficiency and energy balance. The performance evaluation was done by simulated the algorithm with sensors deployed in square area.

COordination-based data Dissemination protocol for wireless sEnsor networks (CODE) is proposed which is based on GAF protocol in [15]. To obtain better results for energy consumption an efficient data dissemination path is formed. This is designed for mobile sinks. Three phases defined in CODE are as Data announcement, Query transfer and Data dissemination.

\section{Conclusion}

WSNs have seen great developments in designing. This progress resulted in solving the problems of this area. Energy efficiency is the basic challenge to overcome in the field of WSN. Different routing protocols have been proposed to reduce the energy consumption. This paper focuses on the energy aware Geographic Adaptive Fidelity (GAF) routing protocol. GAF is highly scalable and saves the energy of nodes by turning the radio off. Only necessary nodes participate in transmission others enter into sleep mode. This paper also gives detailed view about some other researches which are performed on the basis of GAF. Other GAF based protocols are proposed to overcome some issues of GAF and to increase the lifetime of network. Improved versions of GAF gives better performance in comparison to original GAF.

\section{References}

[1] Ridha Soua and Pascale Minet, “A Survey on Energy Efficient Techniques in Wireless Sensor Networks", IEEE Wireless and Mobile Networking Conference (WMNC), pp. 1-9, October 2011

[2] Pallavi Jindal and Vikas Gupta, "Study of Energy Efficient Routing Protocols of Wireless Sensor Networks and Their Further Researches: A Survey”, International Journal of Computer Science and Communication Engineering, Volume 2, Issue 2, pp. 57-62, May 2013.

[3] Sinchan Roychowdhury and Chiranjib Patra, "Geographic Adaptive Fidelity and Geographic Energy Aware Routing in Ad Hoc Routing", Special Issue of International Journal of Computer and Communication Technology (IJCCT), Volume 1, Issue 2, pp. 309313, August 2010.

[4] Gayatri Prajapati, Palak Parmar, "A Survey on Routing Protocols of Location Aware and Data Centric Routing Protocols in Wireless Sensor Network", International Journal of Science and Research (IJSR), India, Volume 2, Issue 1, pp. 128-133, January 2013.

[5] Parul Tyagi and Surbhi Jain, "Comparative Study of Routing Protocols in Wireless Sensor Network", International Journal of Advanced Research in Computer Science and Software Engineering Research Paper, Volume 2, Issue 9, pp.134-140, September 2012.

[6] Tokuya Inagaki and Susumu Ishihara, "HGAF: A Power Saving Scheme for Wireless Sensor Networks", Information Processing Society of Japan Journal, Volume 50, Issue 10, pp. 2520-2531, October 2009.

[7] Susumu Matsumae and Fukuhito Ooshita, "Hierarchical Low Power Consumption Technique with Location Information for Sensor Networks", International Journal of Advanced Computer Science and Applications (IJACSA), Volume 4, Issue 4, pp. 69-74, December 2013. 
[8] QI Xiao-gang and QIU Chen-xi, “An Improvement of GAF for Lifetime Elongation in Wireless Sensor Networks”, IEEE International Conference on Wireless Communications, Networking and Mobile Computing, pp. 1-4, September 2009.

[9] Jiaxi You, Dominik Lieckfeldt, Jakob Salzmann and Dirk Timmermann, "GAF\&Co: Connectivity Aware Topology Management for Sensor Network", IEEE International Symposium on Personal, Indoor and Mobile Radio Communications, pp. 2260-2264, September 2009 .

[10] Ankita K. Patel and Radhika D. Joshi, "Energy Conservation for Wireless Mobile Ad hoc Networks using Hexagonal GAF Protocol", International Conference on Networking, VLSI and Signal Processing (ICNVS), ISSN: 1790-5117, pp. 29-34, February 2010.

[11] Arpan Roy, Adway Mitra, Arijit Khan, and Debashis Saha, “Buffering Techniques in Sleep Doze Coordination and Grid Based Clustering Protocols as Power Management Schemes for Wireless Sensor Networks”, IEEE International Conference on Wireless, Mobile and Multimedia Networks, pp. 240-245, January 2008.

[12] Arpan Roy, Adway Mitra, Arijit Khan, Mita Nasipuri and Debashis Saha, "LSDC A Lossless Approach to Lifetime Maximization in Wireless Sensor Networks", IEEE Sensors Applications Symposium (SAS), pp. 166-171, February 2008.

[13] Abrar Alajlan, Benjamin Dasari, Zyad Nossire, Khaled Elleithy and Varun Pande, "Topology Management in Wireless Sensor Networks: Multi-State Algorithms", International Journal of Wireless \& Mobile Networks (IJWMN), Volume 4, No. 6, pp. 17-25, December 2012.

[14] Guofang Nan, Guanxiong Shi, Zhifei Mao and Minqiang Li, "CDSWS: Coverage-Guaranteed Distributed Sleep/ Wake Scheduling For Wireless Sensor Networks", EURASIP Journal on Wireless Communications and Networking, February 2012.

[15] Hung Le Xuan, Dae Hong Seo, Sungyoung Lee, and Young-Koo Lee, "Minimum-Energy Data Dissemination in Coordinationbased Sensor Networks", IEEE International Conference on Embedded and Real-Time Computing Systems and Applications, pp. 381-386, August 2005. 